\title{
A Novel Measure of Fractional Anisotropy Based on the Tensor Distribution Function
}

\author{
Liang Zhan ${ }^{1, *}$, Alex D. Leow ${ }^{1,2, *}$, Siwei Zhu ${ }^{3}$, Marina Barysheva ${ }^{1}$, \\ Arthur W. Toga ${ }^{1}$, Katie L. McMahon ${ }^{4}$, Greig I. de Zubicaray ${ }^{4}$, \\ Margaret J. Wright ${ }^{5}$, and Paul M. Thompson ${ }^{1}$ \\ ${ }^{1}$ Laboratory of Neuroimaging, Dept. of Neurology, University of California, Los Angeles, \\ USA \\ ${ }^{2}$ Department of Psychiatry, University of Illinois Medical Center at Chicago, USA \\ ${ }^{3}$ Dept. of Mathematics, University of California, Los Angeles, USA \\ ${ }^{4}$ Functional MRI Laboratory, Centre for Magnetic Resonance, University of Queensland, Bris- \\ bane, Australia \\ ${ }^{5}$ Queensland Institute of Medical Research, Brisbane, Australia
}

\begin{abstract}
Fractional anisotropy (FA), a very widely used measure of fiber integrity based on diffusion tensor imaging (DTI), is a problematic concept as it is influenced by several quantities including the number of dominant fiber directions within each voxel, each fiber's anisotropy, and partial volume effects from neighboring gray matter. With High-angular resolution diffusion imaging (HARDI) and the tensor distribution function (TDF), one can reconstruct multiple underlying fibers per voxel and their individual anisotropy measures by representing the diffusion profile as a probabilistic mixture of tensors. We found that FA, when compared with TDF-derived anisotropy measures, correlates poorly with individual fiber anisotropy, and may sub-optimally detect disease processes that affect myelination. By contrast, mean diffusivity (MD) as defined in standard DTI appears to be more accurate. Overall, we argue that novel measures derived from the TDF approach may yield more sensitive and accurate information than DTI-derived measures.
\end{abstract}

\section{Introduction}

Diffusion-weighted MRI is a powerful tool to study water diffusion in tissue, providing vital information on white matter microstructure, such as fiber connectivity and integrity in the healthy and diseased brain. To date, most clinical studies still employ the diffusion tensor imaging (DTI) model [1,2], which describes the anisotropy of water diffusion in tissues by estimating, from a set of $K$ diffusion-sensitized images, the $3 \times 3$ diffusion tensor (the covariance matrix of a 3-dimensional Gaussian distribution). Seven independent gradients are mathematically sufficient to determine the diffusion tensor, but MRI protocols with higher angular and radial resolutions, such as the high angular resolution diffusion imaging (HARDI) [3] or diffusion spectrum

Zhan and Leow contributed equally (correspondences should be sent to Leow at feuillet@ucla.edu). 
imaging techniques [4], have been proposed to resolve more complex diffusion geometries, such as fiber crossings and intermixing of tracts. These geometries are incorrectly captured by a single-tensor model, as employed in standard DTI.

Among several recent advances in HARDI, the Q-ball imaging technique has been proposed to reconstruct fiber orientation density functions (ODFs) from the raw HARDI signal [3]. Deconvolution methods [5] can also yield mathematically rich models of fiber geometries using probabilistic mixtures of tensors [6], fields of von Mises-Fisher mixtures [7], or higher-order tensors (i.e., 3x3x...x3 tensors) [8,9]. Recent work on stochastic tractography $[5,10]$ also exploits the increased angular detail in HARDI. In most deconvolution-based methods, however, prior assumptions on fibers are usually imposed, e.g., all fiber tracts are forced to have the same anisotropy profile. Leow et al. recently proposed a more flexible approach, the Tensor Distribution Function (TDF) [11] to model fiber crossing in HARDI. Using the calculus of variations, the TDF approach can separate different dominant fiber directions in each voxel and compute their individual eigenvalues.

Much progress has been made in modeling more complex diffusion geometries that a single tensor fails to model, but most clinical studies still rely on simple DTIderived scalar measures. Some of these, such as the trace of the covariance matrix or mean diffusivity (MD) can adequately describe isotropic water diffusion, but this only occurs in the cerebrospinal fluid spaces in the brain. In the white matter, myelinated fibers resist water diffusion orthogonal to the local dominant fiber orientation, and diffusion occurs preferentially along local fiber tracts. In clinical research, white matter fiber integrity is commonly assessed by determining how strongly diffusion is directionally constrained. One common scalar measure of directional diffusion, the fractional anisotropy (FA), is computed from the diffusion tensor's eigenvalues, and quantifies the magnitude of this directional preference. Clinical studies now routinely use FA as an index of white matter integrity, sensitive to white matter deterioration in aging and neurodegenerative diseases [12]. Even so, FA does not truly reflect the multidimensional complexity of the water diffusion profile. Regions with complex fiber-crossing tend to have lower FA values than predominantly unidirectional white matter structures (such as the midline corpus callosum; see Figure 2). However, it is unlikely that each of these crossing fibers in these regions has a true decrease in its integrity when compared to, say, corpus callosum fibers. In this paper, we argue that "white matter integrity", as measured by FA, is somewhat vague and imprecise, and may be greatly improved by using the full diffusion gradient information in HARDI. Factors that influence FA values may include the number of dominant fiber directions in each voxel, the eigenvalues of each of these fibers, partial volume effects from neighboring gray matter, and the non-Gaussianity of water diffusion. By using the TDF approach, which can separate crossing fibers, we examine where FA fails to reflect the underlying diffusion anisotropy.

\section{Methods}

Sixteen volunteers were scanned using a diffusion-sensitized MRI protocol on a Bruker Medspec 4 Tesla MRI scanner, with a transverse electromagnetic (TEM) headcoil. The timing and angular sampling of the diffusion sequence was optimized 
for SNR [13]. The protocol used 94 diffusion-sensitized gradient directions, and 11 baseline scans with no diffusion sensitization (b-value: $1159 \mathrm{~s} / \mathrm{mm}^{2}$; TE/TR: 92.3/8250 ms; FOV=230x230; in-plane resolution: $1.8 \mathrm{mmx} 1.8 \mathrm{~mm}$; 55 x 2mm contiguous slices; acquisition time: 14.5 minutes).

To process the data, a positive definite diffusion tensor was firstly estimated from the raw HARDI signal using the MedINRIA software (http://wwwsop.inria.fr/asclepios/ software/MedINRIA), which projects the tensor manifold to its tangent plane at the origin to avoid negative or zero eigenvalues, which do not correspond to a physical diffusion process. Based on the diffusion tensor eigenvalues $\left(\lambda_{1}\right.$, $\lambda_{2}$, and $\lambda_{3}$ ), the FA (defined in this paper as $\mathrm{FA}^{\mathrm{DTI}}$ to avoid confusion) and MD $\left(\mathrm{MD}^{\mathrm{DTI}}\right)$ may be calculated using Equation 1:

$$
\left\{\begin{array}{l}
F A^{D T I}=\sqrt{\frac{3}{2}\left(\frac{\left(\lambda_{1}-<\lambda>\right)^{2}+\left(\lambda_{2}-<\lambda>\right)^{2}+\left(\lambda_{3}-<\lambda>\right)^{2}}{\lambda_{1}^{2}+\lambda_{2}^{2}+\lambda_{3}^{2}}\right)} \\
M D^{D T I}=<\lambda>=\frac{\lambda_{1}+\lambda_{2}+\lambda_{3}}{3}
\end{array}\right.
$$

Values of $\mathrm{FA}^{\mathrm{DTI}}$ range from 0 (no directional dependence of diffusion) to 1 (diffusion along a single direction). In addition to modeling the HARDI signal with a single tensor, we applied the framework in [11] to the HARDI signal, computing the voxelwise tensor distribution function $P$ - a $p d f$ defined on all physiologically feasible $3 \mathrm{D}$ Gaussian diffusion processes (in the tensor space D) at each voxel that best describes the observed signal. In our current implementation, we assume cylindrical fiber tracts and exclude planar-shaped tensors. Thus, $\lambda_{1} \geq \lambda_{2}=\lambda_{3}$ for each individual tensor in this tensor space. Given any TDF $P$, the number of dominant fibers is estimated by examining the local maxima of the tensor orientation distribution (TOD) $\left(T O D(\theta)=\int_{\lambda} P(D(\lambda, \theta)) d \lambda\right)$. The eigenvalues of each fiber can be calculated by computing their expected values along the principal direction of this fiber. In this paper, we will investigate two sets of eigenvalues, i.e., those of the $1^{\text {st }}$ dominant fiber $\left(\lambda_{1}^{1}, \lambda_{2}^{1}\right)$ and the voxelwise TDF-averaged eigenvalues $\left(\overline{\lambda_{1}}, \overline{\lambda_{2}}\right)$ (Equation 2$)$.

$$
\begin{cases}\lambda_{i}^{1}=\frac{\int P\left(D\left(\theta^{*}, \lambda\right)\right) \lambda_{i} d \lambda}{\int P\left(D\left(\theta^{*}, \lambda\right)\right) d \lambda} \quad\left(i=1,2 \text { and } \theta^{*}=\operatorname{argmax}(\operatorname{TOD}(\theta))\right) \\ \overline{\lambda_{\mathrm{J}}}=\int_{\mathrm{D} \in \mathrm{D}} \mathrm{P}(\mathrm{D}) \lambda_{\mathrm{j}} \mathrm{dD} \quad(\mathrm{j}=1,2)\end{cases}
$$

To obtain an overall assessment of the anisotropy in each voxel using the TDF framework, we propose the differential diffusivity (DD) $\left(D D=\left(\overline{\lambda_{1}}-\overline{\lambda_{2}}\right) /\left(\overline{\lambda_{1}}+\overline{\lambda_{2}}\right)\right.$ ). To compare TDF-derived measures to those from standard DTI, we compute the $1^{\text {st }}$ dominant fiber's FA $\left(\mathrm{FA}^{1}\right)$ and $\mathrm{MD}\left(\mathrm{MD}^{1}\right)$, as well as the TDF-averaged FA (FA ${ }^{\mathrm{TDF}}$ ) and $\mathrm{MD}\left(\mathrm{MD}^{\mathrm{TDF}}\right)$ by using the eigenvalues defined in Eq.(2) .

\section{Results and Discussion}

\subsection{The Relation between DTI-FA and Actual Individual Fiber Anisotropy}

We first assessed whether FA derived from standard DTI (FA ${ }^{\text {DTI }}$ ) is an accurate measure of fiber anisotropy. Even though $\mathrm{FA}^{\mathrm{DTI}}$ is usually intended to measure 
white matter integrity, it in fact measures the compound effect of several factors, including the FA of individual fibers, the number of dominant fiber directions and partial volume effect from gray matter. Many of these will vary when the integrity of the fibers is not impaired, confounding its interpretation. Since any disease process that affects white matter myelination will most likely affect individual fiber anisotropy, we here restrict ourselves to investigating how well FA measures the anisotropy of individual fibers. As the TDF approach can separate multiple dominant fibers in one voxel and can determine their respective eigenvalues, we may answer the above question by investigating how well FA $^{\text {DTI }}$ correlates with the FA

Table 1. Correlations between DTI-derived measures and TDF-derived measures for the whole brain and for voxels with $\mathrm{FA}^{\mathrm{DTI}}>0.2$ (thus mainly white matter voxels). $\mathrm{FA}^{\mathrm{DTI}}$ correlates poorly with actual individual fiber anisotropy computed from the TDF. Compared to FA, MD ${ }^{\mathrm{DTI}}$ correlates better with actual individual MD. All correlations reach statistical significance $(\mathrm{p}<0.001)$.

\begin{tabular}{|c|c|c|c|c|c|c|}
\hline Correlations & $\begin{array}{c}\mathrm{FA}^{\mathrm{DTI}} \\
\text { vs. } \mathrm{FA}^{1}\end{array}$ & $\begin{array}{c}\mathrm{FA}^{\mathrm{DTI}} \\
v s . \mathrm{FA}^{\mathrm{TDF}}\end{array}$ & $\begin{array}{c}\mathrm{FA}^{\mathrm{TDF}} \\
\text { vs. } \mathrm{FA}^{1}\end{array}$ & $\begin{array}{c}\text { MD }^{\text {DTI }} \\
\text { vs. } \text { MD }^{1} \\
\end{array}$ & $\begin{array}{c}\text { MD }^{\mathrm{DTI}} \\
\text { vs. } \mathrm{MD}^{\mathrm{TDF}}\end{array}$ & $\begin{array}{c}\text { MD }^{\mathrm{TDF}} \\
\text { vs. MD }\end{array}$ \\
\hline Whole brain & 0.309 & 0.433 & 0.884 & 0.546 & 0.620 & 0.849 \\
\hline Voxels with $\mathrm{FA}^{\mathrm{DTI}}>0.2$ & 0.155 & 0.285 & 0.827 & 0.440 & 0.509 & 0.821 \\
\hline
\end{tabular}

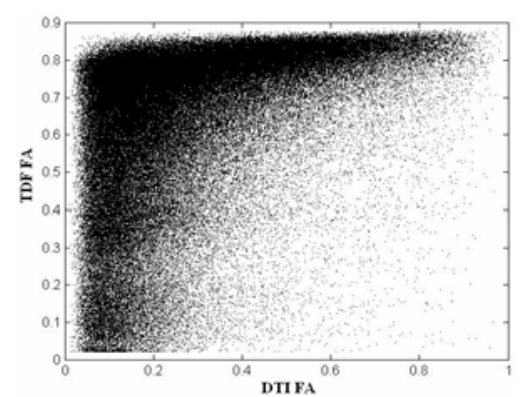

(a) DTI FA vs. TDF FA

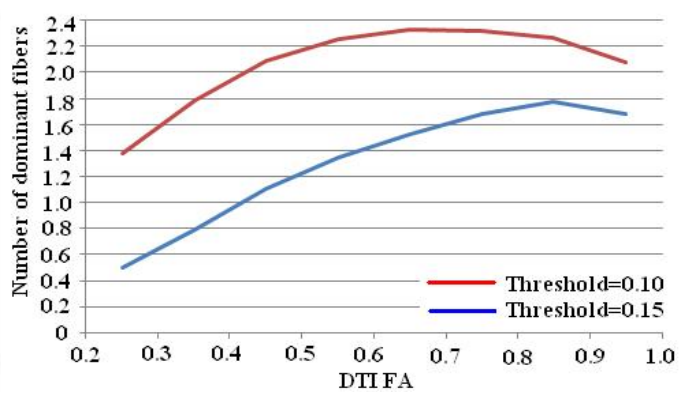

(b) DTI FA vs. Number of dominant fibers

Fig. 1. Comparison between TDF and DTI. (a) Correlation between DTI FA and TDF FA. Here we plotted DTI-derived FA against TDF-averaged FA for the whole brain using one control subject as described in the methods section. FA derived from DTI is highly variable; if this measure does not accurately reflect the anisotropy of the component fibers, statistical power will be sacrificed when investigating group differences in white matter integrity using standard statistical testing (e.g., 2-sample $t$ tests or multiple regressions). (b) DTI FA vs. Estimated number of dominant fibers. FA ${ }^{\mathrm{DTI}}$ values are related to the number of dominant fiber directions, determined by thresholding the corresponding TOD at two different values $(0.15$ and 0.1 ) and counting the number of local maxima (e.g., at a threshold of $0.15,61.2 \%$ of white matter voxels have one dominant fiber direction, $31.6 \%$ have two, and $7.2 \%$ have three or more). The mean number of dominant fiber directions for different ranges of $F A^{\mathrm{DTI}}$ is calculated by averaging the number of fiber directions within that range (e.g., when FA ${ }^{\mathrm{DTI}}$ is within 0.6 and 0.7, the mean number of dominant fiber directions is 1.529). Both curves showed a non-linear relationship - with an initial increase for the number of fiber directions, followed by a decrease as $\mathrm{FA}^{\mathrm{DTI}}$ increases. 
values of the underlying dominant white matter fibers. As more than one fiber direction may be present, we examined how well $\mathrm{FA}^{\mathrm{DTI}}$ correlates with either $\mathrm{FA}^{1}$ or the overall $\mathrm{FA}^{\mathrm{TDF}}$. Table 1 shows the results of these correlations. Only moderate correlations are found $\left(0.471\right.$ for $\mathrm{FA}^{\mathrm{DTI}}$ vs. $\mathrm{FA}^{\mathrm{TDF}}, 0.352$ for $\mathrm{FA}^{\mathrm{DTI}}$ vs. $\left.\mathrm{FA}^{1}\right)$ when both gray and white matter are included. Correlations are much weaker $(0.303$ and 0.164. respectively) when we only consider the white matter (operationally defined by a threshold of FA ${ }^{\mathrm{DTI}}$ at 0.2 ), which is the tissue type FA was originally designed to investigate $\left(\mathrm{FA}^{\mathrm{TDF}}\right.$ correlates well with $\left.\mathrm{FA}^{1}\right)$. By contrast, correlations are better between DTI- and TDF-derived MD measures (Table 1) than for FA (e.g., 0.631 for $\mathrm{MD}^{\mathrm{DTI}}$ vs. $\mathrm{MD}^{\mathrm{TDF}}$ in the whole brain). These results support our hypothesis that in the white matter, $\mathrm{FA}^{\mathrm{DTI}}$ correlates very poorly with the actual individual fiber anisotropy, and thus (1) may be suboptimal for detecting subtle disease processes that affect myelination, and (2) may even be misleading, as low FA values may simply reflect the presence of multiple fibers.

To visualize these correlations, we plotted $\mathrm{FA}^{\mathrm{DTI}}$ against $\mathrm{FA}^{\mathrm{TDF}}$ in Figure 1(a). Visual inspection suggests an overall trend of positive correlation between these two measures, but a closer look suggests that $\mathrm{FA}^{\mathrm{DTI}}$ is highly variable in the white matter. Indeed, for voxels with at least one dominant direction, the mean value of $\mathrm{FA}^{\mathrm{DTI}}$ is 0.39 with a standard deviation (std) of 0.20 , while those for $\mathrm{FA}^{\mathrm{TDF}}$ are 0.74 and 0.16 .

The observation that FA derived from DTI has a higher variability in the white matter is important for clinical applications where group comparisons are often conducted using a two-sample t test, or the general linear model. In these studies, the larger standard deviation of $\mathrm{FA}^{\mathrm{DTI}}$ inevitably lowers the statistical power of a test with a fixed $\mathrm{N}$ (requiring more subjects to detect subtle changes). This further suggests that $\mathrm{FA}^{\mathrm{DTI}}$ is a suboptimal measure of the underlying white matter myelination.

\subsection{The Relation between FA ${ }^{\text {DTI }}$ and the Number of Dominant Fiber Directions}

In the previous section, we showed that $\mathrm{FA}^{\mathrm{DTI}}$ does not reflect the actual anisotropy of the underlying component fibers. In this section, we further explore the relation between $\mathrm{FA}^{\mathrm{DTI}}$ and the number of dominant fibers. Here, we determine the number of dominant fibers by first thresholding the TOD at two different values $(0.1$ and 0.15$)$ and counting the TOD local maxima exceeding these thresholds. As DTI is a singletensor model, one would assume that the correlations with individual fiber measures would decrease (be less accurate) as the number of dominant fibers increases. This is indeed the case for MD (Table 2). However, the same analysis on FA (Table 2) did not reveal a similar trend. Instead, here the correlations first increase (with the number of dominant fibers) then decrease. To understand this discrepancy, we plotted the number of dominant fibers against $\mathrm{FA}^{\mathrm{DTI}}$ in Figure 1(b), which reveals a similarly complex picture. Indeed, the number of dominant fibers first shows an increase when


understand this result, we hypothesize that the highest $\mathrm{FA}^{\mathrm{DTI}}$ values usually correspond to predominantly uni-directional white matter structures (e.g., corpus callosum). These tend to be very heavily myelinated and have fewer dominant fiber directions compared to white matter voxels with fiber-crossing (thus lower $\mathrm{FA}^{\text {DTI }}$ values). However, at low FA $^{\text {DTI }}$ values, we have to consider at least two opposite factors, both of which tend to cause a decrease in $\mathrm{FA}^{\mathrm{DTI}}$ (but opposite trends for the number of 
Table 2. Correlations between DTI-derived measures and TDF-derived measures for different numbers of dominant fibers

\begin{tabular}{|c|c|c|c|c|}
\hline Correlations & \multicolumn{2}{|c|}{$\mathrm{MD}^{\mathrm{DTI}}$ vs. $\mathrm{MD}^{\mathrm{TDF}}$} & \multicolumn{2}{c|}{$\mathrm{FA}^{\mathrm{DTI}}$ vs. FA ${ }^{\mathrm{TDF}}$} \\
\hline Dominant fibers & Whole brain & Voxels with $\mathrm{FA}^{\mathrm{DTI}}>0.2$ & Whole brain & Voxels with $\mathrm{FA}^{\mathrm{DTI}}>0.2$ \\
\hline Number=0 & 0.532 & 0.523 & 0.269 & 0.131 \\
\hline Number=1 & 0.445 & 0.421 & 0.308 & 0.160 \\
\hline Number=2 & 0.377 & 0.361 & 0.314 & 0.213 \\
\hline Number $\geq 3$ & 0.317 & 0.307 & 0.298 & 0.211 \\
\hline
\end{tabular}

fiber directions): the partial volume effect from gray matter (causing a decrease in the number of dominant fibers as the volume ratio of gray matter increases), and the fiber-crossing effect (causing an increase in number of dominant fibers as more fibers cross one another). The combination of these factors may explain the positive correlation of $\mathrm{FA}^{\mathrm{DTI}}$ and the number of dominant fiber directions for low-to-medium FA ${ }^{\mathrm{DTI}}$. Our results are also consistent with those in [14], where FA was positively correlated with fiber density index (which measures how many fibers go through a given voxel) in patients with glioblastoma.

\subsection{The Roles of Differential Diffusivity and Exponential Isotropy in Visualization}

Here, we note that the TDF-derived differential diffusivity (DD), similar to FA, has higher intensities in the white matter than in the gray matter. However, unlike FA ${ }^{\text {DTI }}$,



(a) $\mathrm{FA}^{\mathrm{DTI}}$

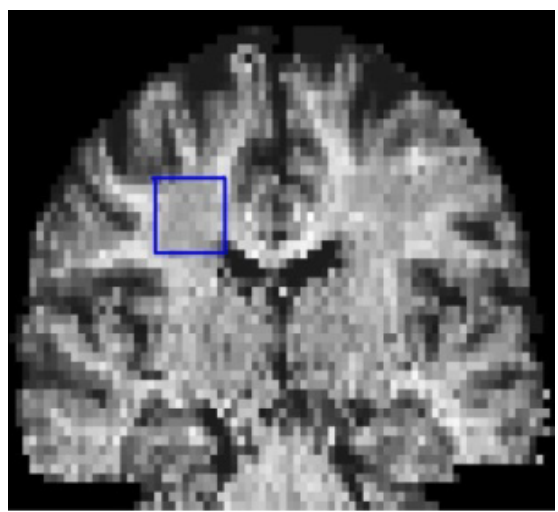

(b) DD

Fig. 2. FA ${ }^{\mathrm{DTI}}$ is incorrectly depleted in regions with extensive fiber crossing (blue box, inset). By contrast, the differential diffusivity (DD), derived using the TDF approach, separates the dominant fiber directions and their corresponding anisotropy measures. In the highlighted region, fiber-crossing is present (fibers of the corpus callosum mix with the corona radiata, and superior longitudinal fasciculus). Here $\mathrm{FA}^{\mathrm{DTI}}$ values are lowered relative to those of neighboring white matter, For DD, the signal is more consistent with that of the fibers entering the highlighted region. 


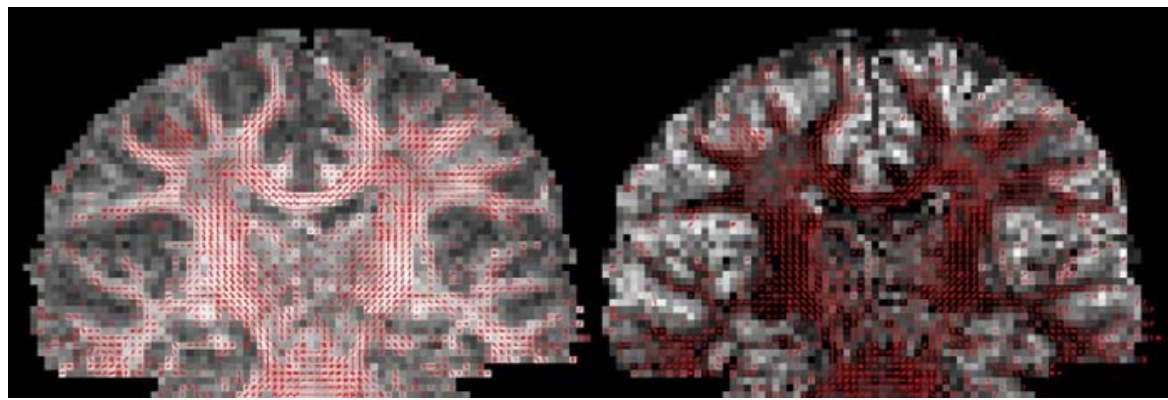

(a)TOD overlaid with $\mathrm{FA}^{\mathrm{DTI}}$

(b) TOD overlaid with EI

Fig. 3. Here, EI quantifies the overall isotropy of any given voxel, and highlights the gray matter instead of the white matter as in FA. EI, when overlaid on fiber directional information such as the TOD, may help visualizing HARDI data, offering information complementary to the FA map. Visually, the EI-TOD plot (right panel) provides a good representation of the spatial configurations of fiber tracts connecting with neighboring gray matter. Notice that in this figure, the TOD plot nicely resolves the fiber crossing in the Pons.

it does not suffer much from a drop in values in voxels with fiber-crossing (Figure 2). Figure 3 shows that, compared to FA, EI appears to help in visualizing fiber orientation mappings such as the TOD plot, as it complements the information in the TOD, and visually provides a good representation of the spatial configurations of fiber tracts connecting with the neighboring gray matter. Notice that in this figure, the TOD plot nicely resolves fiber-crossing in the Pons.

\section{Conclusion}

In this paper, we showed that white matter integrity, measured using the fractional anisotropy (FA) derived from standard DTI, is imprecise as it depends on several quantities including (among others) the number of dominant fiber directions, the anisotropy of each component fiber tract, and partial volume effects from neighboring gray matter. When compared with TDF-derived anisotropy measures, the FA obtained from standard DTI does not correlate well with the actual anisotropy of the individual component fibers, and may be sub-optimal in detecting subtle disease processes that affect white matter myelination. Future imaging studies of white matter integrity may benefit from assessing the number of dominant fiber directions in each voxel, and their corresponding eigenvalues and anisotropy. The TDF framework is ideal for achieving these goals. Lastly, we also demonstrated that the exponential isotropy (EI), differential diffusivity (DD) and the tensor orientation distribution (TOD) (all novel concepts derived from TDF) may help in visualizing HARDI data, as they provide additional information complementary to measures obtained from standard DTI. 


\section{References}

1. Basser, P.J., Pierpaoli, C.: Microstructural and physiological features of tissues elucidated by quantitative diffusion tensor MRI. J. Magn. Reson. B 111(3), 209-219 (1996)

2. Le Bihan, D.: IVIM method measures diffusion and perfusion. Diagn Imaging (San Franc) 12(6), 133-136 (1990)

3. Tuch, D.S.: Q-Ball Imaging. Magnetic Resonance in Medicine 52, 1358-1372 (2004)

4. Tuch, D., Diffusion, M.R.I.: of complex tissue structure. PhD thesis, Harvard UniversityMassachusetts Institute of Technology, Cambridge, Massachusetts (2002)

5. Alexander, D.: Maximum entropy spherical deconvolution for diffusion MRI. In: Proceedings of the 19th International Conference on Information Processing in Medical Imaging (IPMI), Glenwood Springs, CO, USA (2005)

6. Hess, C.P., Mukherjee, P., Han, E.T., Xu, D., Vigneron, D.B.: Q-ball reconstruction of multimodal fiber orientations using the spherical harmonic basis. Magn. Reson. Med. 56, 104-117 (2006)

7. Jansons, K.M., Alexander, D.: Persistent angular structure: new insights from diffusion magnetic resonance imaging data. Inverse Probl. 19, 1031-1046 (2003)

8. Anderson, A.: Measurement of fiber orientation distributions using high angular resolution diffusion imaging. Magn. Reson. Med. 54, 1194-1206 (2005)

9. Özarslan, E., Shepherd, T., Vemuri, B.C., Blackband, S., Mareci, T.: Resolution of complex tissue microarchitecture using the diffusion orientation transform (DOT). NeuroImage 31(3), 1083-1106 (2006)

10. Tournier, J.D., Calamante, F., Gadian, D., Connelly, A.: Direct estimation of the fiber orientation density function from diffusion-weighted MRI data using spherical deconvolution. NeuroImage 23, 1176-1185 (2004)

11. Leow, A.D., Zhu, S., Zhan, L., de Zubicaray, G.I., Meredith, M., Wright, M., Toga, A.W., Thompson, P.M.: The Tensor Distribution Function. Magn Reson Med. 18; 61(1), 205214 (2009)

12. Zhang, Y., Schuff, N., Jahng, G.H., Bayne, W., Mori, S., Schad, L., Mueller, S., Du, A.T., Kramer, J.H., Yaffe, K., Chui, H., Jagust, W.J., Miller, B.L., Weiner, M.: Diffusion tensor imaging of cingulum fibers in mild cognitive impairment and Alzheimer disease. Neurology 68(1), 13-19 (2007)

13. Jones, D.K., Horsfield, M.A., Simmons, A.: Optimal strategies for measuring diffusion in anisotropic systems by magnetic resonance Imaging. Magn. Res. Med. 42(3), 515-525 (1999)

14. Roberts, T.P.L., Liu, F., Kassner, A., Mori, S., Guha, A.: Fiber Density Index Correlates with Reduced Fractional Anisotropy in White Matter of Patients with Glioblastoma. AJNR Am J. Neuroradiol. 26, 2183-2186 (2005) 